\title{
Non-invasive Detection of Human Body Liquor Intake Based on Optical Biosensor
}

\author{
Christian Jhoart Abaoag ${ }^{1}$, Ralph Alforque ${ }^{1}$, John Benedict Ordoño ${ }^{1}$, and Edna Quinto ${ }^{1,2, *}$ \\ ${ }^{1}$ Department of Chemical Engineering, University of Santo Tomas, 1008 Manila, Philippines \\ ${ }^{2}$ Research Center for the Natural and Applied Sciences, University of Santo Tomas, 1008 Manila, Philippines
}

\begin{abstract}
Alcohol-related incidents are increasing despite the implementation of RA 10586, the Anti-Drunk or Drugged Driving Act of 2013. Conventionally, blood alcohol content (BAC) is tested by gas chromatography or breathalyzers. This work aims to design and fabricate a paperstrip based sensor for the detection of alcohol using saliva as biomedium. The sensor will act as an alternative alcohol detection platform, which will provide low cost analysis of BAC. Different tests were undergone using p-nitrophenol, PNP, as recognition element, which include stability, repeatability, and sensitivity. In order to establish the change of PNP in the presence of alcohol, a UV-Vis spectrophotometer was used. For stability and repeatability, an RSD of $1.24 \%$ and $4.61 \%$ were obtained, respectively. Furthermore, the sensitivity to alcohol concentration was found to have an $R^{2}$ value equal to 0.987 . For the paper-strip application, $10 \mathrm{~mm} \times 60 \mathrm{~mm}$ optical sensing membrane (OSM) with immobilized PNP was prepared. It is analysed through the use of digital image captured by a smartphone camera. The RGB values are then measured using the ImageJ software application. The stability, repeatability, and sensitivity of biosensor are $1.85 \%$ RSD, $2.23 \%$ RSD, and 0.9731 coefficient of linearity, respectively. This method is a promising alternative to breathalyzer.
\end{abstract}

\section{Introduction}

Being charged with DUI or DWI (Driving Under the Influence or Driving While Intoxicated) is a very serious offense. If you are found guilty, it will significantly impact your ability to drive (with suspended or revoked license), increase your insurance rates, and may even include a fine and jail time. A guilty court verdict can further affect your employment options, financial future, and ability to travel internationally. [1]

Field Sobriety test and chemical blood test are two of the most commonly performed by policemen in order to detect if a person is under the influence of alcohol.

According to Badugu, et. al., blood sampling is impractical especially in today's generation, since other bodily fluids, such as sweat and saliva, give far better results compared to the blood tests. Using the latter fluid, different components of the blood can be determined: uric acid, lactic acid, alcohol concentration, glucose level, etc. [2]

According to Philippine National Police-Highway Patrol Group (PNP-HPG), road accidents are one of the leading causes of death in the Philippines. In 2012, there was an average of 227 road accidents per day according to MMDA. [3] Vehicular accidents associated with drunk driving is steadily increasing in number according to HPG. In 2012, 375 car accidents were recorded nationwide involving drunk drivers; 390 in 2013, and 498 in 2014. [4]

Breathalyzer units were distributed and have been in disposal of officer in NCR, Metro Davao, and Metro Cebu since March 12, 2015. [5] The device uses a platinum fuel cell that reacts specifically to presence of alcohol, and will not be affected by external or environmental factors. It is also already calibrated according to the Philippines' legal blood alcohol content (BAC) limit of $0.05 \%$. For non-pofessional drivers, their BAC should not exceed $0.05 \%$. Professional drivers and drivers of public utility vehicles (PUVs), however, must have no trace of alcohol at all- anything higher than $0 \%$ will be subject to penalty. [6]

To date the Philippine government will need 3,000 breathalyzers if one is to one ratio per precinct is the target as there are 3,000 precincts throughout the country. [7]

To solve this problem, the researchers intend to design a low-cost, portable, user-friendly version of breath analyser by finding a suitable reagent that is capable of detecting quantitatively both the presence and concentration of ethanol present inside the body through human saliva. The presence of ethanol at varying concentration in saliva will reflect different intensities of visible color change on the biosensor.

Corresponding author: ecquinto@gmail.com 
The study would show that the device/method possess repeatability, stability of the signal, sensitivity of the reagent to changes in the alcohol content of the saliva sample. It should show the limit of detection of the reagent and the response time. A real sample would be tested using the biosensor.

The primary goal for this prototype is to develop an alternative to breathalyzers that are currently commercialized in the market by fabricating a low-cost and portable or compact and easy to carry paper-strip based biosensor with higher efficiency and sensitivity. The development of this biosensor will be advantageous to (1) the country's economy, (2) other professionals, and (3) the general public.

The proposed method uses non-invasive sampling, which may be applicable to determine BAC instead of using the conventional method of gas chromatography or breathalyzer using blood and breath as sample, respectively. However, the study will cover only the determination of alcohol in saliva based on the standard reactions of ethanol. It will not include any other organic substances, such as glucose, glycoproteins, amylase, etc., and inorganic substances, i.e. fluoride, calcium ions, and inorganic phosphates, present in saliva nor will it do interference studies.

The presence of alcohol in human body may exist up to very small quantities when compared to other constituents of human body fluids, which include saliva. For this reason, there is a need for an indicator that has the capacity to detect alcohol even in small quantities. Hence, it will serve as the sensing reagent for alcohol.

\section{Preparation of indicator and alcohol standard solutions}

The indicator was prepared according to its solubility to water. $1.6 \mathrm{~g}$ of PNP (RDH, Spain) was dissolved in 100 $\mathrm{mL}$ of distilled water.

Alcohol solutions of standard concentrations were prepared from ethyl alcohol (J.T. Baker, USA). The stock solution is a $0.14 \%(\mathrm{v} / \mathrm{v})$ alcohol solution. Other concentrations of alcohol, $0.02 \%(\mathrm{v} / \mathrm{v})$ to $0.12 \%(\mathrm{v} / \mathrm{v})$, were derived from the stock solution by serial dilution. These alcohol concentrations are such that the limit alcohol percentage, $0.05 \%(\mathrm{v} / \mathrm{v})$, is between the suitable ranges. It is to clearly see the variations of the readings in the upper and lower boundaries of the target concentration.

The reaction of the p-nitrophenol with ethanol produces phenolate. The color change is brought upon by the $\mathrm{pH}$ adjustment carried out by such reaction. The p-nitrophenol is colorless from $\mathrm{pH} 5$ and has the most intense yellow color at $\mathrm{pH}$ 7. [8]

\section{Spectrophotometric analysis of $p$-nitrophenol}

\section{Instrumentation system}

The instrumentation system for the analysis of $\mathrm{p}$ nitrophenol is composed of a personal computer connected to a UV-Vis spectrophotometer, Perkin Elmer LAMBDA $^{\mathrm{TM}} 25$.

The sample was placed inside a cuvette and was then introduced into the spectrophotometer. The UV-Vis spectrophotometer was interfaced with a personal computer for processing. Data acquisition and off-line analysis was performed using Microsoft Excel (Microsoft Corporation, USA). Spectra readings were taken in the visible region. All spectroscopic experiments were performed at room temperature.

\section{Determination of the working wavelength}

Working wavelength was chosen by reading the absorbance of the blank PNP and comparing it with the spectra of a PNP solution with a few drops of $0.14 \%$ ethanol solution.

The absorbance readings obtained in the two conditions were plotted against the wavelength range considered. The two resulting spectra were compared. The wavelength that gave the largest difference between the two absorbance readings was the working wavelength used in the succeeding sensor tests.

\section{Figures of merit of the sensor}

Stability determines the invariability of the sensor against other atmospheric elements. Ideally, the sensor should not react with other atmospheric elements or compounds other than the target analyte. Signal stability was determined by reading and plotting the absorbance with time. Signal stability was based on the Relative standard deviation of the Absorbance readings done in 2 hours.

The repeatability test determines precision of the results, this is done by alternately reading the absorbance of the blank solution and that of the blank with $0.08 \%$ ethanol.

The sensitivity test determines the response of the PNP to varying alcoholic concentrations. Increasing concentrations of alcohol solution was used within the range of $0.04 \%(\mathrm{v} / \mathrm{v})$ to $0.12 \%(\mathrm{v} / \mathrm{v})$ ethanol.

\section{Testing of the PNP immobilized on paper-strip by photo-monitoring}

\section{Immobilization of the reagent}

A paper-strip (Hard Copy, substance: 20/70 gsm, ultrawhite color) of size $10 \mathrm{~mm}$ by $60 \mathrm{~mm}$ was used as matrix where the reagent was immobilized to create an optical sensing membrane for alcohol detection. Immobilization was carried out by dipping in order to equally distribute the reagent on the surface of the paper strip and allowed to dry.

Instrumentation system 
The immobilized p-nitrophenol on paper strip, IPP needs to be photographed under direct lamp light $(3 \mathrm{~W})$ to ensure consistent lighting. A smartphone camera (Samsung A5 2016, 13 MP, f/ 1.9, $28 \mathrm{~mm}$ ) was used to take pictures of the IPP and these images were subjected to treatment using ImageJ, a computer application with extensive photography capabilities, for their red, green, and blue (RGB) measurements. [9]

A section of the photo was selected for treatment. The section was carefully chosen so that there would be no air gaps or misdistribution of the liquid along the surface of the section chosen. The goal is to achieve a uniform distribution of the liquid sample on the chosen section.

The uniformly selected section of each picture gave particular data points corresponding to the intensity of the color. All photographic experiments were performed at room temperature and at a height $13 \mathrm{~cm}$ from the paper to the lens of the camera.

\section{Figures of merit of the IPP}

In the determination of the stability, the IPP was read and results based on RGB measurement. Stability was determined by determining the RSD of the RGB readings. A graph of RGB versus time is plotted.

The response time is the time at which the IPP can sense the presence of ethanol right after the alcohol is introduced to the matrix.

The response time of the biosensor was obtained from the plot of RGB values with and without alcohol against time. The RGB measurements of the paper-strip based biosensor were measured using photographs taken for 20 minutes every 10 seconds.

To determine the degree of repeatability of the sensor, the RGB measurements of the IPP using photographs taken for 1 minutes every 10 seconds. These were subjected to ImageJ data software. The IPP was exposed to equal concentrations of alcohol every trial. Repeatability is presented as RSD value.

To measure the sensitivity of the IPP, it was exposed to different alcohol concentrations. The data obtained are plotted to give a chart of RGB values versus concentration of alcohol.

\section{Testing IPP using real sample}

The IPP was tested on an individual who has taken a single shot of alcoholic beverage. The procedure that was employed was the same as getting the response time of the sensor. The sample saliva was dropped on the IPP

Each trial succeeded in seconds after taking an alcohol shot.

\section{Results and discussion}

\section{Spectrophotometric analysis of p-nitrophenol}

Series of tests was done using the UV-VIS spectrophotometer. The tests include the determination of the working wavelength, stability, response time, repeatability, and sensitivity.

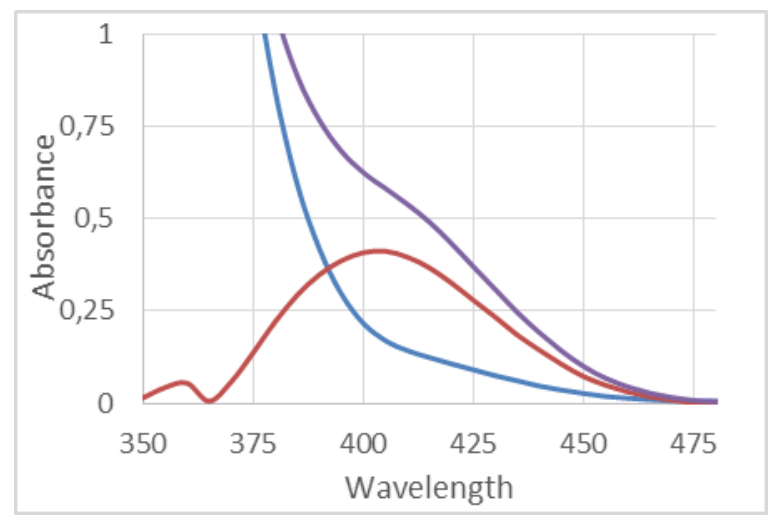

Figure. 1. Spectra of p-nitrophenol in blank medium and $0.14 \%$ ethanol solution

The spectrum in Figure 1 showed the response of pnitrophenol to two different environments: blank (water) and $0.14 \%$ ethanol solution. The third curve is a plot of the difference of the absorbance readings between $0.14 \%$ ethanol concentration and the blank. The result showed that the peak of the curve of the difference occurred at $405 \mathrm{~nm}$. For analytical reading purposes, all readings were done at $405 \mathrm{~nm}$. This is the working wavelength for the succeeding tests.

The stability of p-nitrophenol was a measure of the absorbance for an hour. Analysis of the data resulted to an RSD of $1.24 \%$ This behavior indicated fair stability of the p-nitrophenol at $405 \mathrm{~nm}$ working wavelength.

Figure 2 showed the response time of p-nitrophenol to $0.08 \%$ ethanol solution. Ethanol solution was introduced after taking the absorbance of the blank. There was a significant increase in the absorbance 0.29 seconds after the introduction of ethanol solution.

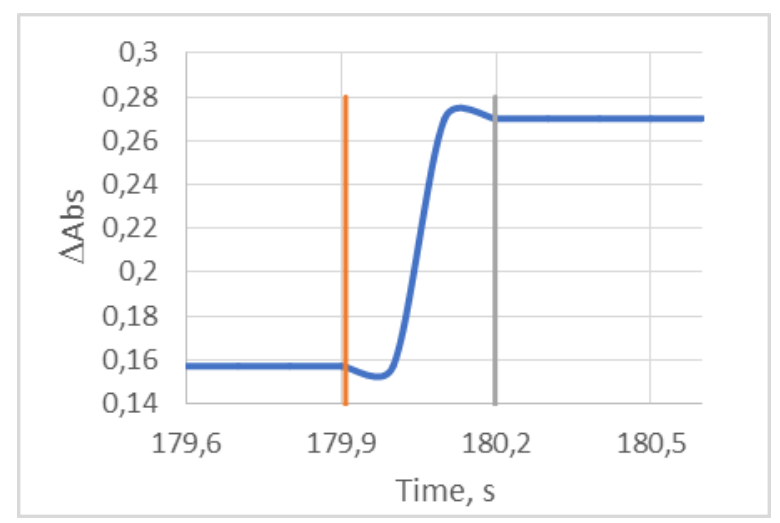

Figure 2. Response time of p-nitrophenol to $0.08 \%$ ethanol solution

Using a $0.08 \%$ solution of ethanol in different runs, repeatability test yielded results shown in Figure 3 with an RSD of $4.61 \%$. 


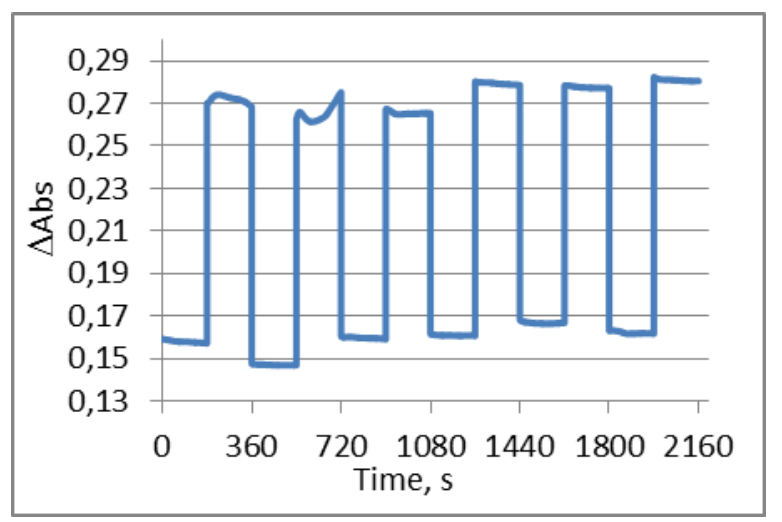

Figure 3. Change in absorbance vs. time using $0.08 \%$ ethanol solution $(\mathrm{n}=6)$

The response of the sensor with varying ethanol concentrations in the range of $0.04-0.1 \%(\mathrm{v} / \mathrm{v})$ is presented in Figure 4. The response showed a direct relationship between change in absorbance and ethanol concentration.

Figure. 4. Sensitivity of the p-nitrophenol to varying ethanol concentration

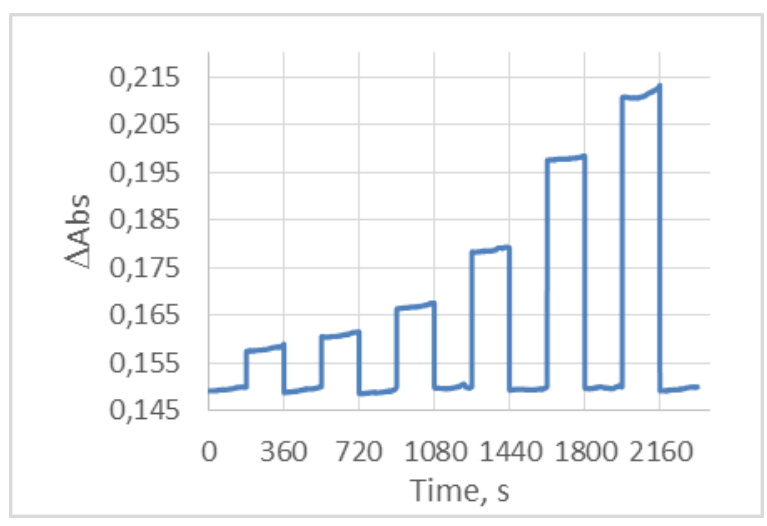

Figure 5 showed the relationship between the change in absorbance readings to the different concentration of alcohol. The sensitivity is 0.56 change in absorbance per $(\mathrm{v} / \mathrm{v}) \%$ ethanol with a coefficient of linearity of $R^{2}=0.987$. The limit of detection of the sensor is $0.02 \%$ ethanol solution.

Figure 5. Calibration curve for the sensitivity of p-nitrophenol indicator $(\mathrm{n}=3)$

\section{Testing of paper-strip biosensor by photo monitoring}

The applicability of the sensor was tested as an immobilized p-nitrophenol using a paper-strip. The same tests were done on the paper-strip which include stability, repeatability, and sensitivity. Using a computer software, ImageJ, the histogram of the solution in the paper was directly observed and analyzed. The RGB (Red, Green, and Blue) color system was utilized and changes in the colors were determined.
Stability is often the most cited parameter in biosensor publications according to Gibson. [10] Furthermore, it relates to the operating lifetime and reusability of the device. The operational stability of a biosensor is defined as the retention of the activity of an indicator when it is immobilized to a medium. The latter investigated if p-nitrophenol, the alcohol indicator, will react to paper, environment, and any other external factors other than the analyte that it should detect. In this experiment, the stability is shown by Figure 6 .

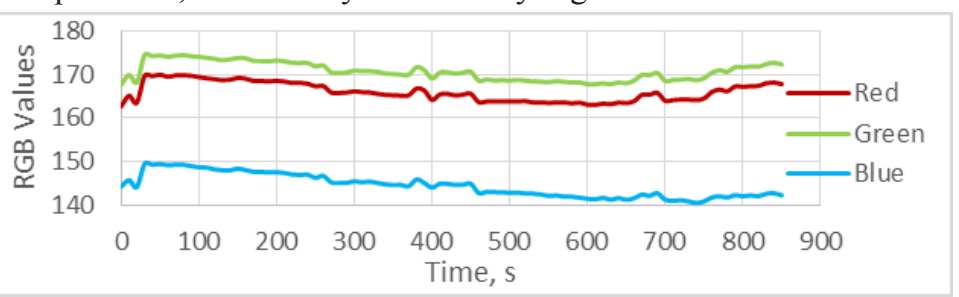

Figure 6. RGB values reading vs. time for stability test

The computed over-all RSD for the signal stability of p-nitrophenol to paper was computed to be $1.31 \%$, $1.23 \%$, and $1.85 \%$ for red, green, and blue respectively. With these RSD values, IPP is stable to paper and can detect the alcohol with a minimal reaction in the environment.

Response time is shown in figure 7. For a $95 \%$ change, $t_{95}$ of the response time is about 270 seconds. In this test, the "blue" color was chosen as the significant indicator.

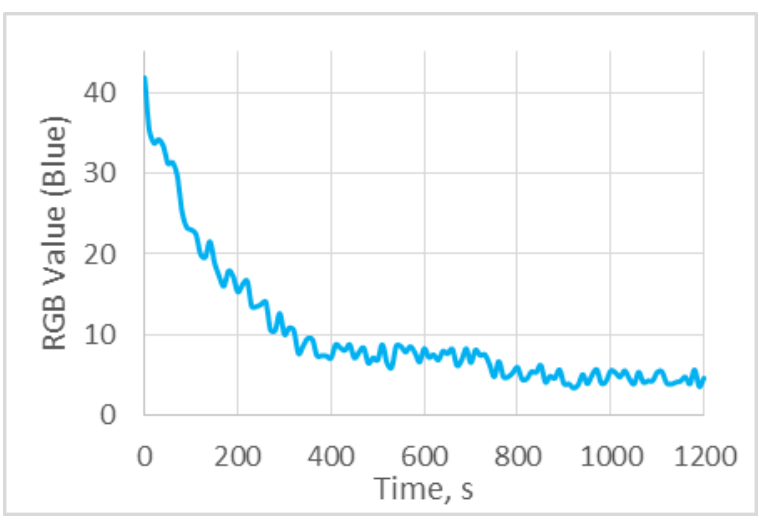

Figure 7. RGB value reading for color blue vs. time

The repeatability of IPP sensor using the same method in the UV-VIS, employed the $0.09 \%$ ethanol. Thiswas prepared based on the $0.05 \%$ minimum allowable ethanol concentration in the human body. The RSD value of the repeatability test is $2.23 \%$. The low RSD value indicates that the biosensor will produce the same result for the same alcohol concentration.

The sensitivity of paper-strip based biosensor is defined by the calibration curve shown in Figure 8. In this test, concentrations of ethanol in the range of 0.04 to $0.1 \%(\mathrm{v} / \mathrm{v})$ were used. Figure 8 showed the reading for color blue. Alcohol concentration of unknown samples can be detected using the calibration curve $y=-191.63 x+118.76$, The actual color response is 
from yellow to lighter or pale yellow when viewed on camera.

As seen in Figure 8, the IPP sensor has a lot to improve. Light scattering and light tightness needs to be improved

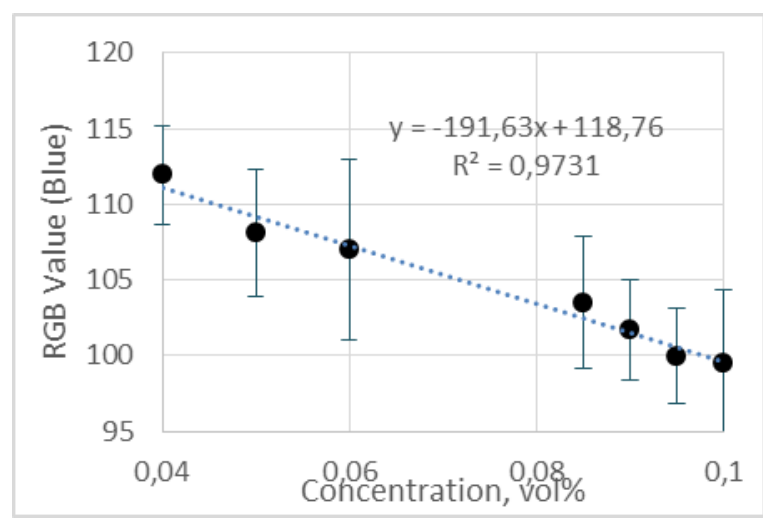

Figure 8. RGB value reading for color blue vs. volume per volume ethanol concentration $(\mathrm{n}=3)$

Limit of detection or (LOD) for this study is obtained by dividing thrice of the standard deviation of the stability data to the slope of the calibration curve. The computed LOD of the biosensor using the color "blue" is $0.03 \%$ per RGB value.

\section{Testing the IPP sensor using real sample}

For the analysis of real sample, six readings were also taken into consideration. Fresh saliva was extracted from the primary source, and immediately tested. Mean of the data from the photo analysis were computed and compared with the calibration curve in order to determine the alcohol concentration. The obtained RGB value for color blue of the real sample is 113.4435. Using the calibration curve formula, the alcohol concentration of the saliva sample was found to be around $0.028 \%(\mathrm{v} / \mathrm{v})$ ethanol. Extrapolation was done as the resulting RGB value is not within the range of the curve.

Based on this limit, the sensitivity of the pnitrophenol biosensor offers a promising detection capability for the quantification of alcohol in human fluid.

\section{Conclusion and recommendation}

In the standardization of the p-nitrophenol as a sensing agent of alcohol, UV-Vis spectrophotometer was used as the main equipment. The absorbance of the solution was utilized in establishing the figures of merit of $\mathrm{p}$ nitrophenol.

The study showed that the reagent is stable when read at $405 \mathrm{~nm}$ with an RSD value of $1.24 \%$ and a response time of 0.29 seconds. The repeatability test showed a $4.61 \%$ RSD. Furthermore, the reagent is sensitive with a linear coefficient of 0.987 and a sensitivity of $0.56 \%$ ethanol/change in absorbance. The limit of detection is $0.02 \%$.

The IPP sensor photographs of the immobilized PNP on paper-strip corresponded to a significant change in RGB values specifically for color "blue". Color changes of the paper-strip from light to intense yellow were observed. The photographs showed a correlation of the response of the paper-strip sensor with alcohol concentration.

The biosensor was analyzed based on its response to alcohol presence. It was found to give a stable signal with an RSD of $1.85 \%$ in the blue region. The response time $t_{95}$ is $270 \mathrm{~s}$. The repeatability test showed an RSD value of $2.23 \%$. The biosensor is also sensitive to different alcohol concentrations. It showed that higher concentrations of alcohol intensify the color of the indicator. Alcohol concentration and RGB values were found to have an inverse relationship of proportionality. The slope of the linear behavior has a sensitivity of 191.63 RGB value per (v/v) \% alcohol. The limit of detection is $0.03 \%$ per RGB value of color blue.

The paper-strip based alcohol biosensor using PNP as an indicator may serve as a low-cost alternative to breathalyzers.

The paper-strip biosensor was tested as an alcohol detector. Further researches on other matrices may be used. Researches on the system response under various conditions such as optimum $\mathrm{pH}$, maybe done to determine the effects on immobilized indicator. Further development may include interference studies since the sample may vary in terms of $\mathrm{pH}$, biological components such as glucose, uric acid, etc.

The paper-strip biosensor may also be improved by using a more controlled environment such as a black box to eliminate other light sources.

Further researches may also include comparison of actual data to those gathered by the breathalyzer and further compare its stability, repeatability and sensitivity to such device to produce a benchmark analysis.

\section{References}

1. Official Gazette of the Republic of the Philippines. (2013, May 27). Republic Act No. 10586. Retrieved December 4, 2016 from the Website of the Official Gazette of the Republic of the Philippines, ttp://www.gov.ph/2013/05/27/republicact-no-10586/

2. G. G. Guilbault, G. Palleschi, G. Lubrano, Biosensors and Bioelectronics, Non-invasive biosensors in clinical analysis, 10.379-392 (1995)

3. Moneymax. (2014, August 10). Top causes of road accidents in the Philippines. Retrieved September 15, 2015, from Moneymax Weblog, $\mathrm{http}: / /$ moneymax.ph/blog/top-causes-roadaccidents-philippines/

4. C.S. Felipe (2015, April 6). Drunk driving cases rising. Retrieved March 28, 2017, from The Philippine Star Website, http://www.philstar.com/metro/2015/04/06/144067 7/drunk-driving-cases-rising 
5. R. Ramirez, M. Frialde (2015, March 7). LTO to start breath analyzer tests next week. Retrieved September 15, 2015, from Philstar Weblog, http://www.philstar.com/metro/2015/03/07/143083 8/lto-start-breath-analyzer-tests-next-week

6. K. Francisco (2015, March 11). Traffic enforcers train in using breathalyzers. Retrieved September 15, 2015, from Rappler Weblog, http://www.rappler.com/nation/86367-lto-breathanalyzer-anti-drunk-driving

7. P. E. Tadeo (2013, July 25). How many breathalyzers will the government need? Retrieved January 25, 2017, from TopGear Philippines Website,http://www.topgear.com.ph/news/motorin g-news/how-many-breathalyzers-will-thegovernment-need

8. Merck Millipore. (2017). 4-Nitrophenol. Retrieved March 28, 2017, from Merck Millipore Website, http://www.merckmillipore.com/INTL/en/product/ 4-Nitrophenol,MDA_CHEM-106798

9. ImageJ. (2016, September 20). ImageJ Ecosystem. Retrieved November 20, 2016, from ImageJ Website, http://imagej.net/Welcome

10. T. D. Gibson, Biosensors, Biosensors: The stability problems, 27 630-638 (1999) 